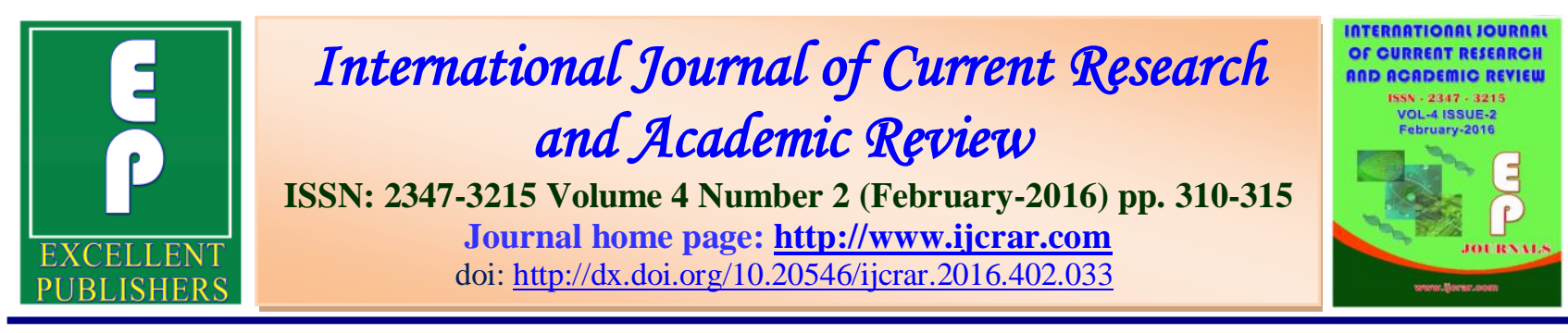

\title{
A Comparative study of Vitamin D in Non ST-Segment elevation myocardial infarction and ST-Segment elevation myocardial infarction patients
}

\author{
M.Shobana $^{1}$, B.Sasi Kumar ${ }^{2}$ and P.Arunachalam ${ }^{3}$ \\ ${ }^{1}$ Junior Resident, Department of Cardiology, Meenakshi Medical College and Research \\ Institute, Kanchipuram-631552, Tamil Nadu, India \\ ${ }^{2}$ Associate Professor, Department of Cardiology, Meenakshi Medical College and Research \\ Institute, Kanchipuram-631552, Tamil Nadu, India \\ ${ }^{3}$ Professor and Head, Department of Cardiology, Meenakshi Medical College and Research \\ Institute, Kanchipuram-631552, Tamil Nadu, India \\ *Corresponding author
}

KEYWORDS

Vitamin D,

ST Segment elevation

Myocardial infarction,

Non ST Segment

elevation

Myocardial

infarction and

Myocardial

Infarction.

\section{A B S T R A C T}

Our recent studies have shown that to investigate the diagnostic role of vitamin D in non ST-segment elevation myocardial infarction and STsegment elevation myocardial infarction patients. Myocardial infarction is the catastrophic frequently fatal form of ischemic heart disease that results from precipitous reduction or arrest of a significant portion of the coronary flow. Vitamin D is a group of fat-soluble secosteroids responsible for intestinal absorption of calcium and phosphate. In humans, the most important related compounds of vitamin D are vitamin D2 and vitamin D3. Acute myocardial infarction was studied in three groups depends on with or without associated complications. in all three groups, mean serum levels of Vitamin D is lower than the mean serum control group. The serum levels of Vitamin D were significantly decreased in STEMI and NSTEMI patients compared with control normal subjects Vitamin D is an useful index, not only in the diagnosis and prognosis, but also in some critical situations of taking some important decision.

\section{Introduction}

Ischemia heart disease is the most widespread health problem over the age 35 . The death rate due to coronary artery disease is increasing in developing countries ${ }^{1}$. The diagnosis and inability to predict prognosis, poses difficult problem, because of a nonavailability of specific and sensitive laboratory tools. The magnitude of this problem dictates, that many medical personnel are involved in some aspects of recognition and treatment of ischemic heart disease, of which myocardial infarction is the most important one ${ }^{2}$.

Once the myocardial infarction has set in, it may take up any of the several pathways. The first and foremost is sudden cardiac death. The incidence of which is $20-25 \%$. If the patient reaches the hospital in a stable 
condition and has no extension of infarction, $75-80 \%$ chances of surviving the attack ${ }^{3}$. However the first one week may be smooth recovery or may be marked by a number of complications ${ }^{4,5}$. Acute myocardial infarction is a type of acute coronary syndrome, which is most frequently a manifestation of coronary artery disease. The acute coronary syndromes include ST segment elevation myocardial infarction (STEMI), non-ST Segment elevation myocardial infarction (NSTEMI).

The possibility of suffering acute myocardial infarction in 40-60years age group is 8times higher than in people of a less advanced age. More than $50 \%$ of in hospital mortality from acute myocardial infarction occurs in subjects older than 65 years ${ }^{4}$.

Vitamin D is a group of fat-soluble secosteroids responsible for intestinal absorption of calcium and phosphate. In humans, the most important related compounds of vitamin D are vitamin D2 and vitamin $\mathrm{D} 3^{6}$. Vitamin $\mathrm{D}$ deficiency continues to be an unrecognized epidemic in many populations around the world ${ }^{6}$. It has been reported in healthy children, young adults, middle-aged adults, and the elderly, and is common among both males and females ${ }^{6}$.

Epidemiological studies indicate an inverse relationship between plasma $25[\mathrm{OH}] \mathrm{D}$ and the occurrence of ST segmental elevation myocardial infarction (STEMI) and Non ST segmental elevation myocardial infarction patient. In UK an increased cardiovascular morbidity is associated with low plasma $25[\mathrm{OH}] \mathrm{D}$ concentrations in winter.

\section{Materials and Methods}

\section{Chemicals}

$25[\mathrm{OH}]$ D kits were purchased from immune Diagnostic kits, USA and all the other chemicals used were of analytical grade.

\section{Experimental Design}

Forty two patients in the age group of 45-70 admitted in the intensive care unit of Meenakshi Medical College Hospital and Research Institute, Kanchipuram, Tamil Nadu for the study. This includes 30 male patients with acute myocardial infarction in whom a provisional diagnosis was made with specific change in electrocardiogram, indicating STEMI patients. The remaining twelve patients, including two females were NSTEMI patient. Patients demographic data, including sex, age, and risk factors for cardiac events including high-risk age (men $>45$, women $>55$ years old), smoking history, medical history of hypertension, hyperlipidemia, diabetes, and a positive family history, drug history, presence of arrhythmia, laboratory data, ECG, and echocardiography findings, were recorded.

The patients were divided into three groups are included

Group-I-Patients were ultimately discharged in good condition (Control)

Group-II-Patients were admitted with ST segmental elevation myocardial infarction Patients (STEMI)

Group-III-Patients were admitted with non ST segmental elevation myocardial infarction Patients (NSTEMI)

\section{Collection of Samples}

Peripheral venous blood $(10 \mathrm{ml})$ samples were collected from each patient upon admission to determine the 25(OH)D serum level. Serum level of $25[\mathrm{OH}]$ D was measured by direct ELISA kit method. In this study, 25(OH)D levels greater than 30 
ng/ml were considered normal; 25(OH)D concentrations of equal to or less than 30 $\mathrm{ng} / \mathrm{ml}$ were considered deficient.

\section{Statistical Analysis}

Data were analyzed using the SPSS software package, version 17.0 (SPSS Inc., Chicago, Illinois, USA). Quantitative data were expressed using range, mean, $\mathrm{SD}$, and median, whereas qualitative data were expressed as frequency and percentage. Qualitative data were analyzed using the $\chi^{2}$ test; also, exact tests such as Fisher's exact were used to compare the two groups. Nonnormally distributed quantitative data were analyzed using the Mann-Whitney test to compare the two groups. The Pearson coefficient was used to analyze the correlation between any two variables. $P$ value was assumed to be statistically significant at 0.05 .

\section{Ethical Concern}

Ethical clearance was obtained from the Ethical committee meeting conducted at Meenakshi Medical College and Hospital.

\section{Results and Discussion}

\section{Presentation According to ECG in Myocardial Infarction Patients}

Table 1 shows the presentation according to ECG in myocardial infarction. This table demonstrates the percentage of STEMI, NSTEMI of male and female in myocardial infarction patients attending Meenakshi Medical College, Enathur, Kanchipuram, India. The percentage of presentation according to ECG with male STEMI, Male NSTEMI and Female NSTEMI levels were respectively $71.4 \%, 23.8 \%$ and $4.76 \% .12$ patients $(28.57 \%)$ presented with NSTEMI symptoms of ECG. In this group, 10 patients were males $(23.8 \%)$ and 2 patients were females $(4.76 \%)$.

\section{Vitamin D in STEMI and NSTEMI Myocardial Infarction Patients}

Fig. 1 represents the study of vitamin D in STEMI and NSTEMI myocardial infarction patients. Group- II STEMI myocardial Infarction patients shows a significantly $(p<0.001)$ decreased when compared with Group I normal control subjects. In Group II NSTEMI myocardial infarction patients were significantly $(p<0.001)$ decreased when compared with normal control GroupI but Group-II STEMI myocardial infarction patients were slightly significantly $(\mathrm{p}=$ 0.701) decreased when compared with Group-III NSTEMI myocardial infarction patients. There was a trend toward lower vitamin D levels with increasing severity of CAD, but the differences were not statistically significant.

MI is myocardial necrosis occurring as a result of critical imbalance between coronary blood supply and myocardial demand. Myocardial infarction is the impairment of heart function due to inadequate blood flow to the heart compared to its need, caused by obstructive changes in the coronary circulation to the heart. In more than $90 \%$ of cases, the cause of myocardial ischemia is reduced blood flow due to obstructive atherosclerotic plaque lesions in one of the three large coronary arteries or its branches. Despite impressive strides in diagnosis and management over the last three decades, acute myocardial infarction continues to be a major public health problem in the industrialized world. Although the death rate of MI has declined by about $30 \%$ over the last decade, its development is still a fatal event in approximately one third of the patients. 
Table.1 Presentation According to ECG in Myocardial Infarction Patients

\begin{tabular}{|c|c|c|}
\hline GENDER & STEMI & NSTEMI \\
\hline MALE & $30(71.4 \%)$ & $10(23.8 \%)$ \\
\hline FEMALE & -- & $2(4.76 \%)$ \\
\hline
\end{tabular}

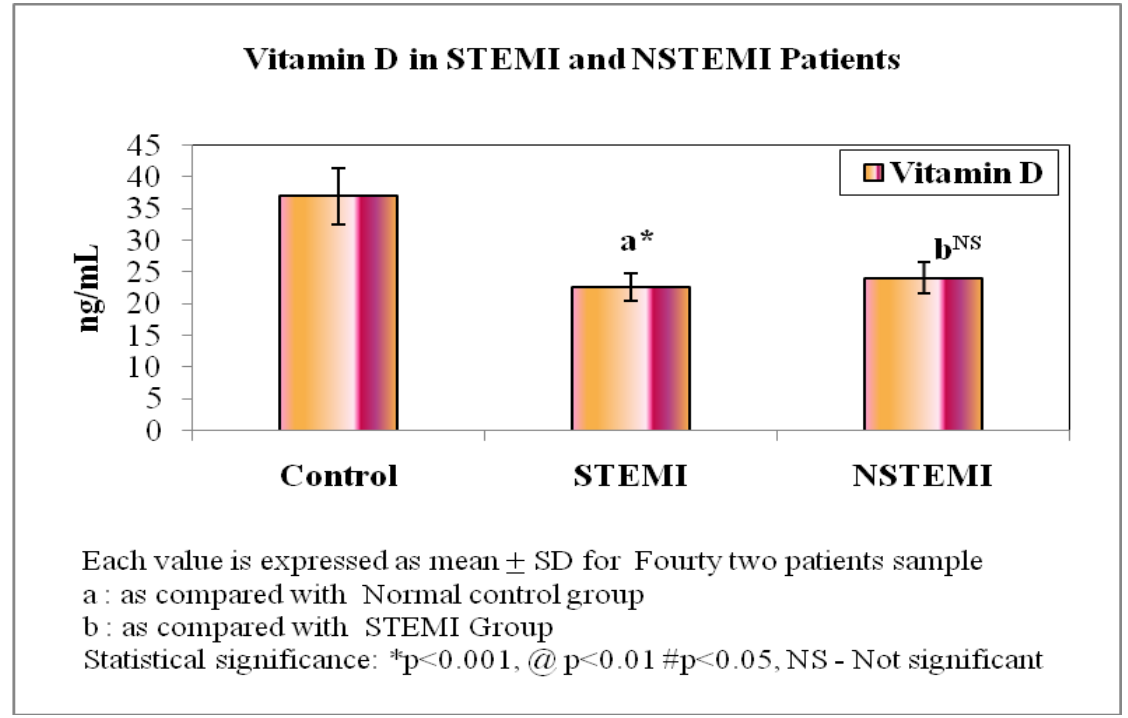

In the present study shows age wise distribution of controls, STEMI and NSTEMI Myocardial Infarction cases. It included healthy controls with mean age of $48.8+5.3$ Years, STEMI Myocardial Infarction cases with mean age of $56.7+6.2$ years and NSTEMI Myocardial infarction cases with mean age of $57.1+6.3$ years.

In patients with STEMI male and female percentage in the present study was $(66 \%$ and 50\%) was comparable with Mutlu et al (67\% and 51.2\%) and L. Newby et al (66\% and $50.2 \%$ ). In patients with NESTEMI, males and female percentage in the present study was $26.6 \%$ and $57.1 \%$ comparable with Muellar et al ${ }^{7}$.

The present study, aimed at investigating the role of vitamin $\mathrm{D}$ in a consective, nonselected cohort of STEMI and NSTEMI patients. Multiple lines of evidence suggest a link between Vitamin D and cardiovascular disease. Epidemiological studies reported that the rates of coronary artery disease, dibetes, hypertension, as well as of vitamin $\mathrm{d}$ deficiency, increase in proportion to increasing distance from the equator ${ }^{8}$. Cardiac death has also been reported to be at its highest during periods of decreased sunlight exposure. Moreover, observational studies, small clinical trials, and meta- analyses indicate that vitamin D therapy may reduce cardiovascular events and mortality. 9,10 Clinical interest derives from the fact that vitamin D deficiency can be readily determined by blood testing and treated by supplementation. In particular, a single oral ultra - high dose of vitamin $\mathrm{D}$ has been shown to restore normal $25(\mathrm{OH}) \mathrm{D}$ levels within 2 daysin critically ill patients, without causing adverse effects. Khalili $\mathrm{H}$ et $\mathrm{al}^{11}$ observed that Vitamin D levels were significantly decreased in Myocardial infarction patients

VDR has also been demonstrated in heart muscle cells and $1,25(\mathrm{OH})_{2} \mathrm{D}$ may play a role in the maintenance of ventricular pump function. Patients with heart failure have 
lower plasma levels of $25(\mathrm{OH}) \mathrm{D}$ and $1,25(\mathrm{OH})_{2} \mathrm{D}$ than controls ${ }^{12}$. There is growing evidence that atherosclerosis may be viewed as a chronic inflammatory disease that involves tumour necrosis factor alpha (TNF- $\alpha$ ) and interleukin-6(IL-6). Active vitamin $\mathrm{D}\left[1,25(\mathrm{OH})_{2} \mathrm{D}\right]$ can suppress these cytokines in vitro and TNF- $\alpha$ is inversely related to plasma $25(\mathrm{OH}) \mathrm{D}$ in vivo ${ }^{13,14}$. Epidemiological studies indicate an inverse relationship between plasma $25(\mathrm{OH}) \mathrm{D}$ and the occurrence of acute myocardial infarction (AMI) ${ }^{15}$. In UK an increased cardiovascular morbidity is associated with low plasma $25(\mathrm{OH}) \mathrm{D}$ concentrations in winter.

\section{Conclusion}

From the present study, estimation of serum vitamin $\mathrm{D}$ is an useful index, not only in the diagnosis and prognosis, but also in some critical situations of taking some important decisions like assessment of condition of the patients for transfer from coronary unit or for discharge. Vitamin D levels were significantly lower in patients with STEMI and NSTEMI Patients compared with controls. Patients with lower levels of vitamin $\mathrm{D}$ had higher prevalence of severe disease on coronary angiography. Further studies with a larger number of patients are warranted to confirm our results.

\section{References}

1.Jadhav PP, Taskar AP, Taskar SP, Darne RD. Evaluation of apo lipoprotein A1 and Apo B in survivors of myocardial infarction. JAPI 1994; 42(9): 703-705.

2.Allison RB, Rodriguez FL, and Higgins EA. Clinicopathological correlation in coronary atherosclerosis. Four hundred thirty patient studies with postmortem coronary angiography. Circulation 1963:27:170.
3.K.Park.Epidemiology of chronic non communicable diseases and conditions. In:Park'sTextbook of Preventive and Social Medicine. 20th ed. Jabalpur: M/S Banarasidas Bhanot Publishers. 2009:315-345

4.Gonzalo Suarez et al. Prediction on admission of in hospital mortality in patients older than 70 yrs with acute myocardial infarction.American College of Chest Physicians.1995: 108:83-88.

5.Holay MP, Janbandhu A, Javahirani A, Pandharipande MS, Suryawanshi SD. Clinical profile of acute myocardial infarction in elderly (prospective study)JAPI Vol. 55 Mar2007; 188-192.

6.Holick MF, DelucaHF, Avioli LV, Isolation and identification of 25hydroxycholecalciferol from human plasma. Archives of Internal Medicine.
1972, 129
(1): 56-61.

7.Muller R T, Gould CA, Betzu R, Vachek T, Pradeep V. Painless myocardial infarction in the elderly. Am Heart J.1990; 119:202-203.

8. Pittas AG, Chung M, Trikalinos T, Mitri J, Brendel M, patel K, et al. Systematic review: vitamin $\mathrm{D}$ and cardio metabolic outcomes. Ann intern Med 2010; 152:307-314.

9.Holick MF. Vitamin D deficiency. N Engl J Med 2007; 357:266-281.

10.Thomas MK, Lioyd - Jones DM, Thadhani RI, Shaw AC, Drraska DJ, Kitch BT. Hypovitaminosis D in medical in patients. N Engl JMed 1998; 338:777-783.

11.Khalili H, talasaz AH, Salarifar M. serum vitamin $\mathrm{D}$ concentration status and its correlation with early biomarkers of remodeling following acute myocardial infarction. Clin Res cardiol 2012; 101:321-327. 
12. Melamed ML, Michos ED, Post W, Astor B. 25-Hydroxyvitamin D levels and the risk of mortality in the general population. Arch Intern Med 2008; 168:1629-1637.

13.Helard L, Mateus-Hamdan L, Beauchet O, Annweiler C. Hypovitaminosis D in geriatric acute care unit: a biomarker of longer length of stay. Dis Markers 2013; 35:525-529.

14.Peterlik M, Cross HS. Vitamin D and calcium insufficiency-related chronic diseases: molecular and cellular pathophysiology. Eur J Clin Nutr 2009; 63:1377-1386.

15. Mahdavi K, Amirajam Z, Yazdankhah S, Majidi S, Adel MH, Omidvar B, Alasti M. The prevalence and prognostic role of vitamin D deficiency in patients with acute coronary syndrome: a single centre study in South-West of Iran. Heart Lung Circ 2013; 22:346-351.

\section{How to cite this article:}

Shobana, M., B. Sasi Kumar and Arunachalam, P. 2016. A Comparative study of Vitamin D in Non ST-Segment elevation myocardial infarction and ST-Segment elevation myocardial infarction patients. Int.J.Curr.Res.Aca.Rev. 2016.4(2): 310-315. doi: http://dx.doi.org/10.20546/ijcrar.2016.402.033 